\title{
Genetic Engineering and Therapy for Inherited and Acquired Cardiomyopathies
}

\author{
SHARLENE DAY, ${ }^{a}$ JENNIFER DAVIS,${ }^{b}$ MARGARET WESTFALL $, b, c$ \\ AND JOSEPH METZGER ${ }^{c}$ \\ ${ }^{a}$ Department of Internal Medicine, University of Michigan, Ann Arbor Michigan, \\ 48103, USA \\ ${ }^{b}$ Department of Molecular and Integrative Physiology, University of Michigan, \\ Ann Arbor Michigan, 48103, USA \\ ${ }^{c}$ Department of Surgery, University of Michigan, Ann Arbor Michigan, 48103, \\ USA
}

\begin{abstract}
The cardiac myofilaments consist of a highly ordered assembly of proteins that collectively generate force in a calcium-dependent manner. Defects in myofilament function and its regulation have been implicated in various forms of acquired and inherited human heart disease. For example, during cardiac ischemia, cardiac myocyte contractile performance is dramatically downregulated due in part to a reduced sensitivity of the myofilaments to calcium under acidic pH conditions. Over the last several years, the thin filament regulatory protein, troponin $I$, has been identified as an important mediator of this response. Mutations in troponin $I$ and other sarcomere genes are also linked to several distinct inherited cardiomyopathic phenotypes, including hypertrophic, dilated, and restrictive cardiomyopathies. With the cardiac sarcomere emerging as a central player for such a diverse array of human heart diseases, genetic-based strategies that target the myofilament will likely have broad therapeutic potential. The development of safe vector systems for efficient gene delivery will be a critical hurdle to overcome before these types of therapies can be successfully applied. Nonetheless, studies focusing on the principles of acute genetic engineering of the sarcomere hold value as they lay the essential foundation on which to build potential gene-based therapies for heart disease.
\end{abstract}

KEYWORDS: myofilament regulation; troponin I; gene delivery; sarcomere; gene-based therapies

Address for correspondence: Joseph M. Metzger, Department of Molecular and Integrative Physiology and Internal Medicine, University of Michigan, 1301 E. Catherine St., Ann Arbor MI 48109-0622. Voice: 734-647-6460; fax: 734-647-6461.

e-mail: metzgerj@umich.edu

Ann. N.Y. Acad. Sci. 1080: 437-450 (2006). (C) 2006 New York Academy of Sciences. doi: 10.1196/annals.1380.033 


\section{INTRODUCTION}

Heart disease claims more lives in the United States each year than the next four leading causes of death combined, accounting for one death every $35 \mathrm{s.}{ }^{1}$ Coronary heart disease is the most prevalent form of cardiovascular disease, affecting 13 million Americans and claiming the lives of $40 \%$ of those who experience a coronary ischemic event in a given year. ${ }^{1}$ Heart failure is similarly a highly prevalent and fatal condition that affects 5 million Americans and carries a 1 -year mortality rate of $20 \%$. These are staggering statistics, particularly in view of the considerable advances that have been made in medical, device, and surgical therapies for heart disease in the last decade. Clearly there is a continued need to develop novel therapies for cardiovascular diseases that go beyond contemporary medicine. Some investigators contend that we may have reached somewhat of a plateau with conventional treatments and predict a shift in emphasis toward gene-based therapy. ${ }^{2}$

Designing molecular therapies that target the ischemic or failing heart requires an understanding of the mechanisms that govern myocardial contractility and relaxation under both normal and pathological conditions. Acute myocardial ischemia alters key aspects of cardiac cell metabolism that, in turn, result in impaired contractility. Prominent among these is intracellular acidification caused by lactate formation during anaerobic glycolysis and by hydrolysis of high-energy phosphate compounds. ${ }^{3}$ When blood flow to the myocardium is acutely compromised, cardiac muscle intracellular $\mathrm{pH}$ drops precipitously (from 7.0 to $\sim 6.2$ ), directly depressing myocardial force ${ }^{4}$ (FIG. 1 A). This
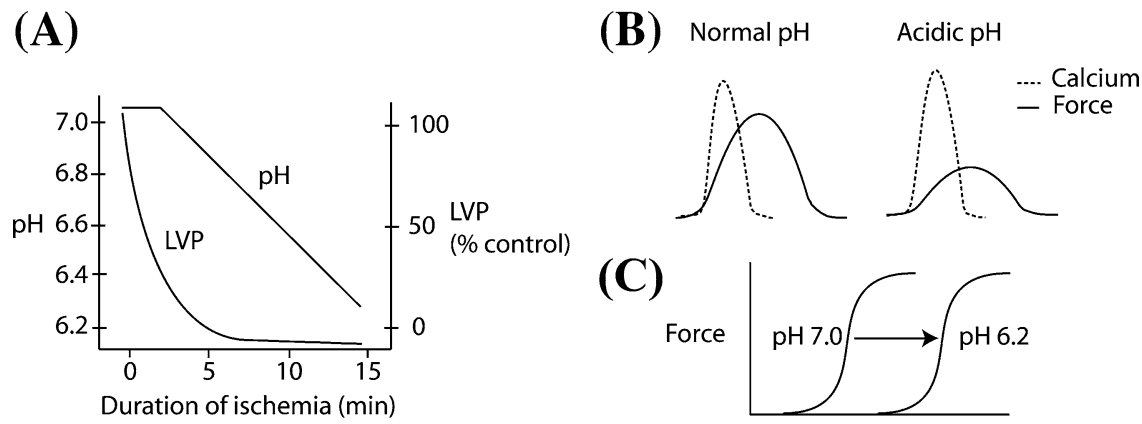

(C)

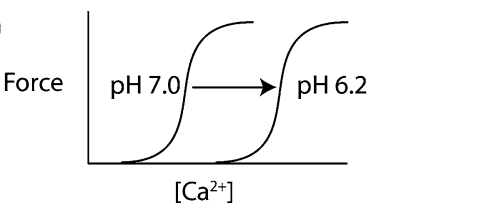

FIGURE 1. Effects of acidosis on force and calcium. (A) Graphic representation of the precipitous drop in both left ventricular pressure and $\mathrm{pH}$ during cardiac ischemia. Adapted from Orchard and Kentish 1990. Am. J. Physiol. 258: C967-C981. (B) Effects of acidic pH on force and the $\mathrm{Ca}^{2+}$ transient in adult cardiac muscle. Force declines when intracellular $\mathrm{pH}$ drops, but the $\mathrm{Ca}^{2+}$ transient remains unchanged or may even increase. Thus, there is an uncoupling of excitation and contraction. (C) Acidic $\mathrm{pH}$ desensitizes the myofilaments to calcium, resulting in a marked rightward shift in the force- $\mathrm{Ca}^{2+}$ relationship. 
depression in contractility occurs despite an unchanged or even increased intracellular $\mathrm{Ca}^{2+}$ transient, indicating a disruption of normal excitationcontraction coupling (FIG. $1 \mathrm{~B}$ ). The basis for this phenomenon is an uncoupling of the response of the contractile proteins to activating calcium. This is manifest in a marked rightward shift in the force- $\mathrm{Ca}^{2+}$ relationship under acidic conditions (FIG. 1 C). In adult cardiac preparations, the $\mathrm{pCa}\left(-\log \left[\mathrm{Ca}^{2+}\right]\right)$ required for $50 \%$ maximal activation falls by an average of $\sim 0.12 \mathrm{pCa}$ units for each 0.1 unit drop in $\mathrm{pH}^{5}{ }^{5}$ In contrast, the contractile function of neonatal heart muscle is less affected than adult myocardium by acidic $\mathrm{pH}, \sim 0.04 \mathrm{pCa}$ unit per 0.1 unit drop in $\mathrm{pH}^{5-7}$ Developmentally regulated transitions in the isoform expression profile for the myofilament protein troponin I (TnI) correlate with shifts in the $\mathrm{pH}$-mediated alteration in the force- $\mathrm{Ca}^{2+}$ relationship noted for neonatal and adult myocardium. ${ }^{8}$ Thus, elucidation of key structural and functional characteristics of TnI isoforms could form the basis for new molecular therapies to improve contractile function during cardiac ischemia.

The importance of the myofilaments in regulation of cardiac function is further underscored by elucidation of the genetic underpinnings of a broad spectrum of cardiac muscle diseases over the past two decades. Primary inherited cardiomyopathies represent an increasingly recognized cause of "idiopathic" heart failure and are phenotypically categorized into three distinct clinical subtypes: hypertrophic (HCM), dilated (DCM), and restrictive (RCM) cardiomyopathies. While clinical classification of these cardiomyopathies is based predominantly on morphologic and hemodynamic characteristics, many structurally divergent cardiomyopathies share common genetic loci. Mutations in genes encoding contractile, cytoskeletal, calcium cycling, and metabolic proteins have been associated with a variety of cardiomyopathic phenotypes. Of these, sarcomeric gene mutations are the most prevalent and well characterized to date, with over 11 genes identified as causal for multiple cardiomyopathic subtypes $^{9}$ (FIG. 2). Since the anatomic and functional changes associated with primary cardiomyopathic diseases are also observed in acquired forms of heart failure (i.e., chronic ischemia, hypertension, diabetes, or valvular disorders), it is likely that altered sarcomere function plays an important role in the pathogenesis of acquired cardiomyopathies as well. Therefore, genetic or molecular strategies that target the sarcomere would be expected to have broad therapeutic benefit.

\section{TnI ISOFORMS AND ACIDOSIS}

TnI is the inhibitory subunit of the troponin complex that toggles between actin (diastole) and troponin $\mathrm{C}$ (systole) in a $\mathrm{Ca}^{2+}$-dependent manner within the sarcomere. ${ }^{10}$ There are two isoforms of TnI that are expressed in the heart at different times during development. Typically, the slow skeletal troponin I isoform (ssTnI) is expressed during the embryonic and early postnatal period. 


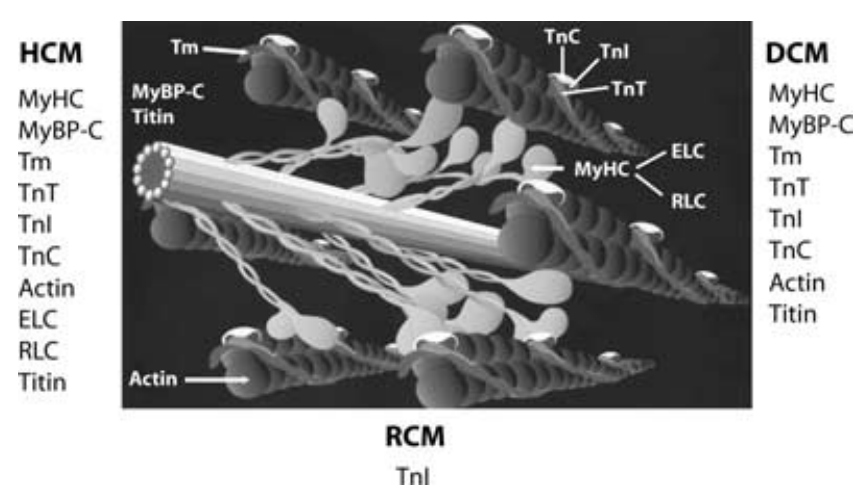

FIGURE 2. Identification and sarcomeric localization of genetic loci harboring mutations linked to inherited cardiomyopathies.

Shortly after birth, there is a transition in expression to the cardiac isoform (cTnI) that persists throughout adult life. ${ }^{11,12}$ This TnI isoform transition correlates with the shift in the force- $\mathrm{Ca}^{2+}$ relationship in response to changes in $\mathrm{pH}^{8}{ }^{8}$ In the adult cTnI-expressing heart, contractility is severely depressed during acidosis, largely due a decrease in the sensitivity of the myofilaments to $\mathrm{Ca}^{2+}$. In contrast, the neonatal ssTnI-expressing heart demonstrates less of a decrease in myofilament $\mathrm{Ca}^{2+}$ sensitivity in an acidic $\mathrm{pH}$ environment compared to the adult heart, and consequently, contractile function is relatively preserved. ${ }^{8}$ Although early studies showed a correlation between TnI isoform expression and myofilament $\mathrm{pH}$ sensitivity, the direct role played by $\mathrm{TnI}$ isoforms in these developmental responses was obscured by concurrent changes in other thin filament proteins over a similar time period.

Genetic engineering of TnI has clearly elucidated its isoform-dependent role in $\mathrm{pH}$-mediated contractile dysfunction. Replacement of cTnI with ssTnI in adult cardiac myocytes using adenoviral-mediated gene transfer results in a marked enhancement of myofilament $\mathrm{Ca}^{2+}$ sensitivity at acidic $\mathrm{pH} .{ }^{6}$ These data are supported by transgenic mouse studies in which replacement of native cTnI with a ssTnI transgene increases papillary muscle tension development during acidosis. ${ }^{13}$ Further studies using chimeras of cTnI and ssTnI provide evidence that this $\mathrm{pH}$ sensing domain localizes to the carboxyl terminus of cTnI. ${ }^{14,15}$ More recently, we have identified a key residue, histidine at position 132 in mouse ssTnI, that uniquely confers acidic $\mathrm{pH}$ resistance of the $\mathrm{Ca}^{2+}$ activated myofilament apparatus. ${ }^{16}$ Adenoviral gene transfer of a modified ssTnI molecule, in which alanine was substituted for histidine at position 132 (H132A), into adult cardiac myocytes converts the ssTnI to the cTnI phenotype in terms of isometric, $\mathrm{Ca}^{2+}$-activated tension at acidic $\mathrm{pH} .{ }^{16}$ Similarly, substituting histidine for alanine at codon 164 in $\mathrm{cTnI}(\mathrm{A} 164 \mathrm{H})$ by gene transfer results in a modest gain of $\mathrm{Ca}^{2+}$-activated tension at neutral $\mathrm{pH}$, an effect that 
is accentuated at acidic $\mathrm{pH} .{ }^{16}$ These data suggest that $\mathrm{TnI}$ isoform dependence of $\mathrm{pH}$ sensitivity may relate to unique biochemical properties of this histidine residue. Owing to its imidazole side chain, histidine is deprotonated and hydrophobic at neutral $\mathrm{pH}$, and protonated and hydrophilic at acidic $\mathrm{pH}$. Thus the positive charge on histidine during intracellular acidosis could weaken interactions between $\mathrm{TnI}$ and arginine or lysine residues in actin, or strengthen interactions with glutamate or aspartate residues in troponin $\mathrm{C}(\mathrm{TnC})$, or both (FIG. 3 A). Any of these possibilities would tend to favor a stronger TnI-TnC association, and thus maintenance of contractile activation under acidic $\mathrm{pH}$ conditions.

\section{ENGINEERING THE SARCOMERE FOR THE ISCHEMIC AND FAILING HEART}

Myocardial ischemia can result in immediate and profound contractile failure, which often persists even after blood flow and intracellular $\mathrm{pH}$ have been restored. Existing pharmacological therapies for heart failure associated with ischemia are limited, often carry undesirable proarrhythmic side effects, and increase energy demand in the already metabolically compromised heart. ${ }^{17,18}$ Therefore, a genetic-based approach focusing on the sarcomere to improve cardiac function during ischemia could offer an attractive alternative.

Intracellular acidosis is a prominent component of cardiac ischemia and contributes to contractile dysfunction and myocellular injury. Direct enhancement of myocardial function under acidic $\mathrm{pH}$ conditions may be advantageous during ischemia. Based on the cellular characterization studies in isolated myocytes, the genetically engineered cardiac $\mathrm{TnI}$ molecule, $\mathrm{A} 164 \mathrm{H}$, appears to be a promising therapeutic candidate to test in the ischemic heart. Indeed, recent studies demonstrate a protective effect of this cTnI variant under a variety of pathophysiological conditions in the whole heart ex vivo and in vivo. ${ }^{16}$ Transgenic mice were generated in which $\sim 80-85 \%$ of native cTnI was stoichiometrically replaced with cTnI A164H. Mouse hearts were retrograde perfused with an acidic buffer in the context of an ex vivo isovolumic heart preparation. Transgenic cTnI A164H mouse hearts were far more resistant to acidosis than nontransgenic controls, demonstrating improved systolic and diastolic performance. In vivo hypoxia and acute coronary ischemia studies yielded similar results; improved cardiac contractility in $\mathrm{cTnI}$ A164H transgenic mice under both conditions. We recently proposed that direct augmentation of sarcomeric $\mathrm{Ca}^{2+}$ activation under acidic $\mathrm{pH}$ conditions could account for the results described here.

During global ischemia and subsequent reperfusion in isolated hearts, cTnI A164H hearts demonstrated markedly improved myocardial recovery, attributable to both better contractility and a blunting of postischemic contracture. ${ }^{16}$ In addition, transgenic replacement with $\mathrm{cTnI}$ A164H was associated 

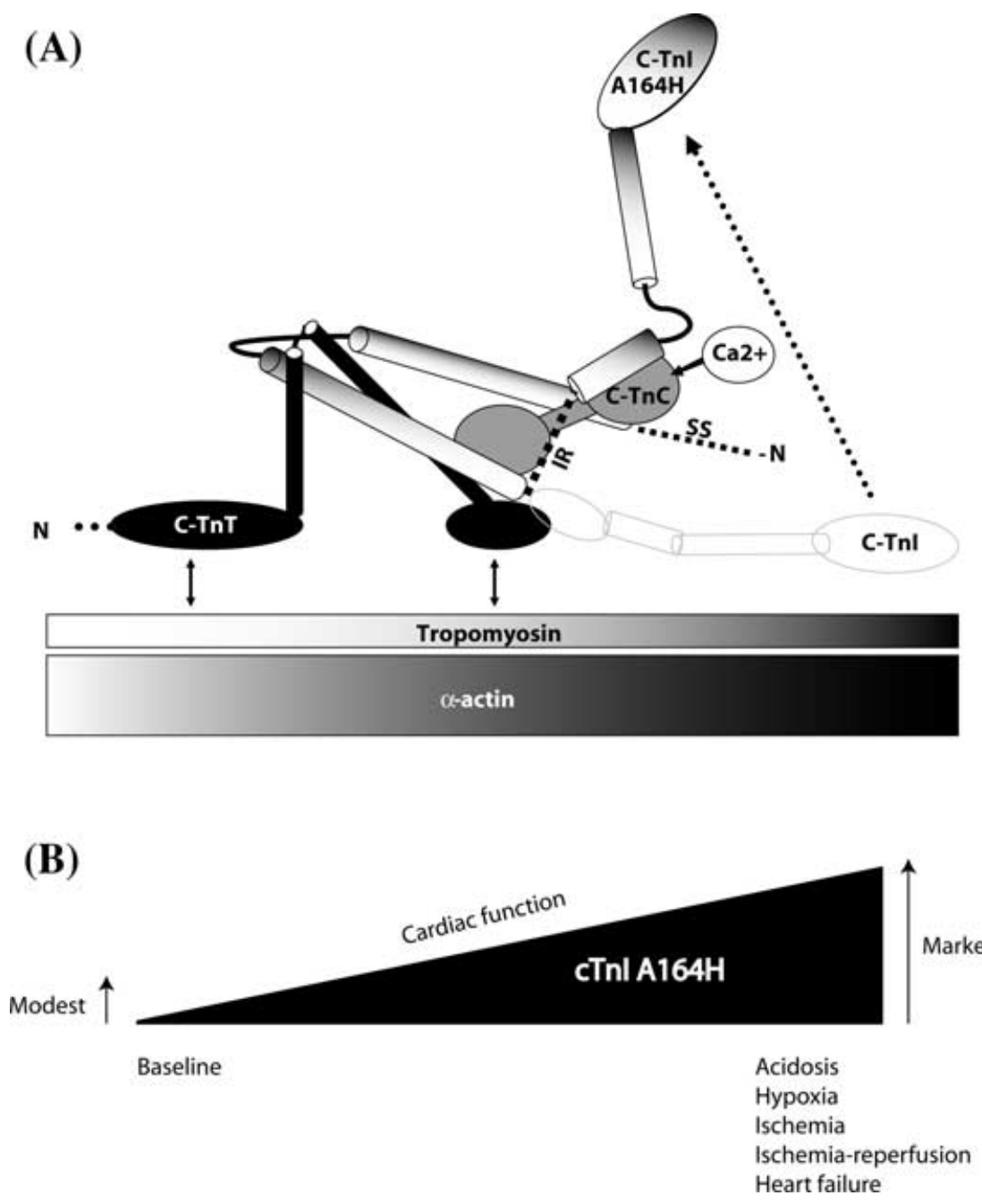

FIGURE 3. Proposed biochemical and functional effects of cTnI A164H. (A) Schematic representation of the troponin complex and its interactions based on the troponin core crystal structure of Maeda and colleagues (TAKedA S., A. Yamashita, K. MAEDA \& Y. MAEDA. 2003. Structure of the core domain of human cardiac troponin in the $\mathrm{Ca}(\mathrm{z}+)-$ saturated form. Nature 424: 35-41.). Cardiac troponin I (cTnI) A164H may stabilize the open confirmation, through strengthened interactions with $\mathrm{TnC}$ or weakened interactions with actin, or both. These effects are predicted to be greatest at acidic $\mathrm{pH}$ when histidine is protonated and hydrophilic. (B) Summary of the effects of TnI A164H replacement on cardiac function. The observed improvement in cardiac performance is most pronounced under pathophysiological conditions. 
with a significant decrease in ventricular arrhythmias. These beneficial effects of $\mathrm{cTnI} \mathrm{A} 164 \mathrm{H}$ in the postischemic heart were surprising, given that $\mathrm{pH}$ is restored quite rapidly after the onset of reperfusion. Potential $\mathrm{pH}$-independent mechanisms to explain improved cardiac function during reperfusion include better recovery of energetic substrates, or attenuation of the $\mathrm{Ca}^{2+}$ overload state characteristic of the postischemic heart. ${ }^{31} \mathrm{P}-\mathrm{NMR}$ spectroscopy studies provide evidence that $\mathrm{cTnI} \mathrm{A} 164 \mathrm{H}$ hearts may be operating in a more economical manner in terms of high-energy phosphate utilization, both under baseline conditions and during reperfusion. Furthermore, cTnI A164H hearts demonstrated important adaptations in $\mathrm{Ca}^{2+}$ handling that may have occurred by virtue of a reduced requirement for intracellular activating $\mathrm{Ca}^{2+}$. Collectively, these changes may contribute to improving performance in cTnI A164H transgenic hearts during ischemia-reperfusion. ${ }^{16}$

The A164H substitution in cTnI was also associated with an augmentation in the function of failing mouse hearts after myocardial infarction. Transgenic mice expressing cTnI A164H demonstrated improved systolic function and an attenuated ventricular remodeling and hypertrophic response. Additionally, adenoviral gene transfer of cTnI A164H into failing human cardiac myocytes obtained at the time of cardiac transplantation improved contractility and relaxation and restored a more favorable force-frequency response. Taken together with the above experimental results demonstrating the beneficial effects of the cTnI A164H protein in the acute settings of acidosis, hypoxia, ischemia, and ischemia-reperfusion, these data support the concept that genetic engineering of the sarcomere could offer a viable strategy for treating ischemic heart disease and heart failure. Sarcomere gene-based therapy represents a promising new approach for redressing heart disease, and could stand alone, or perhaps complement other emerging genetic therapies targeting signaling pathways or calcium homeostasis. ${ }^{19}$

\section{SARCOMERE DYSFUNCTION IN INHERITED CARDIOMYOPATHIES}

\section{HCM}

HCM is the most prevalent inherited cardiovascular disorder, affecting 1 in 500 individuals. ${ }^{20} \mathrm{HCM}$ carries an overall annual mortality rate of $1 \%$, but accounts for over a quarter of sudden cardiac deaths in young competitive athletes. ${ }^{21} \mathrm{HCM}$ is phenotypically heterogenous, but is generally characterized by increased left ventricular wall thickness, primarily involving the interventricular septum. Dynamic left ventricular outflow tract obstruction occurs in $30-50 \%$ of patients and can exacerbate symptoms of breathlessness and chest pain. $^{22}$ The histopathological hallmarks of HCM include myocyte disarray, myocyte hypertrophy, and interstitial fibrosis. 
Over 400 mutations in 11 different sarcomeric genes have been identified as causal for HCM, with mutations in $\beta$-myosin heavy chain (MYH7) and myosin binding protein $\mathrm{C}(M Y B P C 3)$ being the most prevalent. ${ }^{9}$ Sarcomere mutations are identified in $30-70 \%$ of HCM patients, the frequency of which is highly dependent on the characteristics of the study population and the comprehensive nature of the genetic analysis. ${ }^{23-25}$ The remaining cases may be due to other as yet unidentified mutations in sarcomere protein genes, or to mutations in other nonsarcomeric genes. ${ }^{26}$ While each particular sarcomere gene mutation likely influences the clinical phenotype, establishing genotype-phenotype correlations is confounded by the vast number of mutations and phenotypic diversity of HCM. For example, certain mutations have been associated with a particularly malignant phenotype (MYH7 R403Q and R719W), and others with a more benign clinical course (MYH7 L908V, G256E, and V606M, and several MYBPC 3 mutations). ${ }^{25,27}$ However, these associations are derived largely from pedigree analyses and may overestimate the correlation in more unselected populations. ${ }^{23,24}$ Gene dosage does appear to influence the severity of the HCM phenotype. Patients who are homozygous for a mutation or compound heterozygous for different mutations ( $2-5 \%$ of the HCM population) are diagnosed at a younger age, have more hypertrophy, and carry a poorer prognosis than do patients with only one mutant allele. ${ }^{23,28}$ Other proposed important influences on HCM phenotype include environmental factors and genetic modifiers. ${ }^{25,29}$

Significant advances have been made in elucidating the molecular basis for HCM through the use of a variety of experimental biochemical, cellular, and animal models. Collectively, these models suggest that sarcomere gene mutations act via a dominant negative mechanism resulting in a change of function at the molecular level. Biophysical studies indicate that most HCM-linked mutations directly heighten myofilament $\mathrm{Ca}^{2+}$ sensitivity causing increased force production at lower $\left[\mathrm{Ca}^{2+}\right]_{i}$. The magnitude of change in $\mathrm{Ca}^{2+}$ sensitivity results in a corresponding increase in contractility and an impairment of relaxation in the intact cell. ${ }^{30}$ Increased myofilament $\mathrm{Ca}^{2+}$ sensitivity is the primary cause of slowed relaxation kinetics seen in the unloaded cardiomyocyte, and likely contributes to diastolic dysfunction in the whole heart. While the pathways leading from altered $\mathrm{Ca}^{2+}$ sensitivity to myocardial remodeling are incompletely understood, there is evidence to support mechanisms involving altered $\mathrm{Ca}^{2+}$ homeostasis, ${ }^{31,32}$ remodeling of the cardiac action potential, ${ }^{33}$ increased energetic demands, ${ }^{34,35}$ and activation of hypertrophy signaling pathways. ${ }^{25}$

\section{DCM}

DCM affects fewer individuals ( $~ 1$ in 2800$)$ than HCM, but carries a worse prognosis with only $50 \%$ survival at 5 years after diagnosis. ${ }^{1} \mathrm{DCM}$ is inherited 
in about $35 \%$ of cases, most commonly in an autosomal dominant fashion. ${ }^{25}$ DCM is characterized by ventricular wall thinning and dilation and impaired contractile function. The histopathological findings are often nonspecific, revealing myocyte hypertrophy, myocyte degeneration, and interstitial fibrosis. The first identified mutations linked to DCM were in genes encoding for cytoskeletal proteins. ${ }^{36}$ This led to the hypothesis that the pathogenesis of DCM may be related to impaired force transmission between the contractile apparatus, the extracellular matrix, and the sarcolemmal membrane. However, subsequent identification of mutations in genes ( 25 genetic loci to date) encoding proteins with diverse functions suggested the existence of several pathways that could independently lead to DCM. Seven of the loci linked to DCM encode for sarcomeric proteins actin, $\beta-\mathrm{MHC}, \alpha-\mathrm{MHC}$, titin, cTnT, cardiac troponin $\mathrm{C}(\mathrm{cTnC})$, and $\alpha$-tropomyosin (Tm) ${ }^{9,37,38}$ In recent biochemical studies, DCM-causing mutations in $\mathrm{Tm}$, TnT, and $\mathrm{TnC}$ uniformly result in decreased myofilament $\mathrm{Ca}^{2+}$ sensitivity and depressed myofibrillar function after thin filament reconstitution. ${ }^{39,40}$ These changes are quantitatively opposite to those resulting from HCM-associated thin filament mutations, suggesting that distinct changes in myofilament activation may account, at least in part, for the divergent clinical phenotypes observed in DCM compared to HCM.

\section{RCM}

RCM is the least common inherited cardiomyopathy ( $~ 5 \%$ of all cardiomyopathies) but carries the worst prognosis. ${ }^{25}$ Idiopathic RCM typically presents early in childhood and frequently results in early transplantation or sudden cardiac death. RCM is characterized by decreased myocardial compliance and diastolic dysfunction, but normal to near-normal systolic function and myocardial wall thickness. Extensive interstitial fibrosis is a common histopathological finding. ${ }^{41}$ A recent study identified six different mutations (L144Q, R145W, A171T, K178E, D190G, and R192H) in the cTnI gene that were linked to both RCM and HCM phenotypes within the same families. ${ }^{42}$ Many of these family members presented with early heart failure, and patients with the L144Q, D190G, and R192H cTnI mutations all died of sudden cardiac death before the fourth decade of life. The molecular basis for RCM has not been well characterized, although biochemical studies using reconstituted contractile systems from two different laboratories indicate that RCM mutations in cTnI result in a potent increase in myofilament $\mathrm{Ca}^{2+}$ sensitivity of force generation and ATPase activity. ${ }^{43,44}$ The degree of myofilament $\mathrm{Ca}^{2+}$ hypersensitivity resulting from these cTnI mutations is quantitatively greater than that observed in similar previous experiments using exclusively HCM-causing cTnI mutations (R145G, R145Q, R162W, $\Delta$ K183, S199N, G203S, and K206Q). These data suggest that the magnitude of change in myofilament $\mathrm{Ca}^{2+}$ sensitivity 
may be a stimulus for the differentiation of distinct HCM or RCM pathways. However, the association of the same cTnI mutation with either HCM or RCM within the same family also suggests that additional genetic or environmental factors are important in modifying the disease phenotype.

\section{GENETIC ENGINEERING FOR INHERITED CARDIOMYOPATHIES}

There are a number of genetic therapies for heart failure that have been tested in a variety of animal models, most targeting components of calcium handling or the adrenergic signaling cascade. ${ }^{19,45,46}$ Interruption of hypertrophic signaling pathways has also been achieved in animal models of pressure overload hypertrophy. ${ }^{47-49}$ Similarly directed sarcomeric-based therapies may also remediate the functional and structural remodeling processes associated with inherited cardiomyopathies. The sarcomere maintains stoichiometric proportions between each protein constituent. Introduction of an exogenous gene encoding a sarcomere protein into an individual myocyte in vitro, or into the whole heart in vivo, results in stoichiometric replacement rather than overexpression of the contractile protein, such that the total amount of the targeted protein remains unchanged. ${ }^{50,51}$ Endogenous myofilament turnover lays the framework for the development of sarcomere gene transfer approaches for inherited cardiomyopathies. Exogenous expression of a wild-type allele could in principle "compete off" a mutant allele of the same gene, thereby limiting the effective mutant gene dosage. Alternatively, introduction of a functionally complementary sarcomere gene allele might be able to counter the effects of a particular mutant allele on force production or propagation. For example, "normalization" of myofilament $\mathrm{Ca}^{2+}$ sensitivity might be achieved by introducing a sarcomeric variant that heightens $\mathrm{Ca}^{2+}$ sensitivity into a heart expressing a DCM-linked sarcomere mutation associated with reduced $\mathrm{Ca}^{2+}$ sensitivity and vice versa. These sarcomere gene-based therapeutic approaches, while theoretically promising, await further experimental study.

\section{SUMMARY}

The myofilaments play a central role in the regulation of cardiac function in acquired and inherited forms of human heart disease. Contractile dysfunction during cardiac ischemia is directly attributable to an uncoupling of the response of the contractile proteins to activating calcium. TnI is an important mediator of this response, and expression of different $\mathrm{TnI}$ isoforms during cardiac development are largely responsible for the divergent effects of acidic $\mathrm{pH}$ observed in neonatal versus adult myocardium. Recent studies highlight the importance of a single C-terminal amino acid (A164 in cTnI and H132 in ssTnI) in regulating cardiac function during acidosis, ischemia, and heart 
failure. Mutations in genes encoding a number of sarcomere proteins trigger various forms of inherited cardiomyopathies, including hypertrophic, dilated, and restrictive subtypes. Thus, altered sarcomere function forms the pathogenetic basis of a broad spectrum of cardiac muscle diseases, and provides an appealing target for gene-based therapies. The capability of the sarcomere to incorporate exogenous proteins and maintain stoichiometric proportions would facilitate such therapeutic approaches. Further studies focusing on sarcomere genetic engineering in the cell and whole organ, as well as on development of safe and efficient vector systems for gene delivery into the cardiac myocyte in vivo, will lay the foundation for new gene-based therapies for common acquired and inherited forms of heart disease.

\section{ACKNOWLEDGMENTS}

This work was supported by grants from the NIH and the American Heart Association.

\section{REFERENCES}

1. Thом, T., N. HAASe, et al. 2006. Heart disease and stroke statistics-2006 Update. A Report From the American Heart Association Statistics Committee and Stroke Statistics Subcommittee Circulation.

2. MiтK, A.M. 2006. Do lackluster trial findings mean new avenues are needed for heart research? JAMA 295: 611-612.

3. KatZ, A. 2001. Physiology of the Heart. Lippincott Williams and Wilkins. Philadelphia.

4. OrChaRD, C.H. \& J.C. Kentish. 1990. Effects of changes of $\mathrm{pH}$ on the contractile function of cardiac muscle. Am. J. Physiol. 258: C967-C981.

5. Solaro, R.J., J.A. Lee, J.C. Kentish \& D.G. Allen 1988. Effects of acidosis on ventricular muscle from adult and neonatal rats. Circ. Res. 63: 779-787.

6. Westfall, M.V., E.M. Rust \& J.M. Metzger 1997. Slow skeletal troponin I gene transfer, expression, and myofilament incorporation enhances adult cardiac myocyte contractile function. Proc. Natl. Acad. Sci. USA 94: 5444-5449.

7. WestFall, M.V., F.P. Albayya, I.I. Turner \& J.M. MetzGer 2000. Chimera analysis of troponin I domains that influence $\mathrm{Ca}^{2+}$-activated myofilament tension in adult cardiac myocytes. Circ. Res. 86: 470-477.

8. Reiser, P.J., M.V. WeStFall, S. SchiafFino \& R.J. Solaro 1994. Tension production and thin-filament protein isoforms in developing rat myocardium. Am. J. Physiol. 36: H1589-H1596.

9. Nhlbi Program for Genomic application. 2006. Genomics of Cardiovascular Development, Adaptation, and Remodeling. Harvard Medical School. Ref Type: Electronic Citation

10. Westfall, M.V. \& J.M. MetZger 2001. Troponin I isoforms and chimeras: tuning the molecular switch of cardiac contraction. News Physiol. Sci. 16: 278-281.

11. SagGin, L., L. GorZA, S. Ausoni \& S. SchiafFino. 1989. Troponin I switching in the developing heart. J. Biol. Chem. 264: 16299-16302. 
12. HunKeler, N.M., J. Kullman \& A.M. MurPhy. 1991. Troponin I isoform expression in human heart. Circ. Res. 69: 1409-1414.

13. Wolska, B.M., K. Vijayan, G.M. Arteaga, et al. 2001. Expression of slow skeletal troponin $I$ in adult transgenic mouse heart muscle reduces the force decline observed during acidic conditions. J. Physiol. 536: 863-870.

14. Westfall, M.V., I.I. Turner, F.P. Albayya \& J.M. Metzger 2001. Troponin I chimera analysis of the cardiac myofilament tension response to protein kinase A. Am. J. Physiol. 280: C324-C332.

15. Westfall, M.V., F.P. Albayya \& J.M. Metzger 1999. Functional analysis of troponin I regulatory domains in the intact myofilament of adult single cardiac myocytes. J. Biol. Chem. 274: 22508-22516.

16. Day, S.M., M.V. WeStFall, et al. 2006. Histidine button engineered into cardiac troponin I protects the ischemic and failing heart. Nat. Med. 12: 181-189.

17. TeERLINK, J.R. 2005. Overview of randomized clinical trials in acute heart failure syndromes. Am. J. Cardiol. 96: 59G-67G.

18. Bayram, M., L.L. De, M.B. Massie \& M. Gheorghiade. 2005. Reassessment of dobutamine, dopamine, and milrinone in the management of acute heart failure syndromes. Am. J. Cardiol. 96: 47G-58G.

19. HoshiJima, M. 2005. Gene therapy targeted at calcium handling as an approach to the treatment of heart failure. Pharmacol. Ther. 105: 211-228.

20. MARON, B.J. 2002. Hypertrophic cardiomyopathy: a systematic review. JAMA 287: $1308-1320$.

21. Maron, B.J. 2003. Sudden death in young athletes. N. Engl. J. Med. 349: 10641075.

22. Nishimura, R.A. \& D.R. Holmes JR. 2004. Clinical practice. Hypertrophic obstructive cardiomyopathy. N. Engl. J. Med. 350: 1320-1327.

23. Richard, P., P. ChARRON, L. CARRIER, et al. 2003. Hypertrophic cardiomyopathy: distribution of disease genes, spectrum of mutations, and implications for a molecular diagnosis strategy. Circulation 107: 2227-2232.

24. VAn Driest, S.L., S.R. OMmen, et al. 2005. Sarcomeric genotyping in hypertrophic cardiomyopathy. Mayo Clin. Proc. 80: 463-469.

25. Ahmad, F., J.G. Seidman \& C.E. Seidman. 2005. The genetic basis for cardiac remodeling. Annu. Rev. Genomics Hum. Genet. 6: 185-216.

26. Blair, E., C. ReDwood, et al. 2001. Mutations in the gamma(2) subunit of AMPactivated protein kinase cause familial hypertrophic cardiomyopathy: evidence for the central role of energy compromise in disease pathogenesis. Hum. Mol. Genet. 10: $1215-1220$.

27. Charron, P., O. Dubourg, et al. 1998. Clinical features and prognostic implications of familial hypertrophic cardiomyopathy related to the cardiac myosinbinding protein. C Gene Circ. 97: 2230-2236.

28. Ho, C.Y.,H.M. Lever, et al. 2000. Homozygous mutation in cardiac troponin T: implications for hypertrophic cardiomyopathy. Circulation 102: 1950-1955.

29. Marian, A.J. 2002. Modifier genes for hypertrophic cardiomyopathy. Curr. Opin. Cardiol. 17: 242-252.

30. Michele, D.E., C.A. Gomez, K.E. Hong, et al. 2002. Cardiac dysfunction in hypertrophic cardiomyopathy mutant tropomyosin mice is transgene-dependent, hypertrophy-independent, and improved by beta-blockade. Circ. Res. 92: 255262.

31. FAtKin, D., B.K. Mcconnell, et al. 2000. An abnormal Ca(2+) response in mutant sarcomere protein-mediated familial hypertrophic cardiomyopathy. J. Clin. Invest. 106: 1351-1359. 
32. Semsarian, C., I. Ahmad, et al. 2002. The L-type calcium channel inhibitor diltiazem prevents cardiomyopathy in a mouse model. J. Clin. Invest. 109: 10131020 .

33. Knollmann, B.C., P. KirchHof, et al. 2003. Familial hypertrophic cardiomyopathy-linked mutant troponin $\mathrm{T}$ causes stress-induced ventricular tachycardia and $\mathrm{Ca} 2+-$ dependent action potential remodeling. Circ. Res. 92: 428-436.

34. SPINDLER, M., K.W. SAUPE, et al. 1998. Diastolic dysfunction and altered energetics in the alphaMHC403/+ mouse model of familial hypertrophic cardiomyopathy. J. Clin. Invest. 101: 1775-1783.

35. JAVADPOUR, M.M., J.C. TARDIFF, I. PINZ \& J.S. IngWALL 2003. Decreased energetics in murine hearts bearing the R92Q mutation in cardiac troponin T. J. Clin. Invest. 112: $768-775$.

36. FAtKin, D. \& R.M. Graham. 2002. Molecular mechanisms of inherited cardiomyopathies. Physiol. Rev. 82: 945-980.

37. Kamisago, M., S.D. Sharma, et al. 2000. Mutations in sarcomere protein genes as a cause of dilated cardiomyopathy. N. Engl. J. Med. 343: 1688-1696.

38. Daehmlow, S., J. ERdmann, et al. 2002. Novel mutations in sarcomeric protein genes in dilated cardiomyopathy. Biochem. Biophys. Res. Commun. 298: 116120.

39. Chang, A.N., K. Harada, M.J. Ackerman \& J.D. Potter. 2005. Functional consequences of hypertrophic and dilated cardiomyopathy-causing mutations in alphatropomyosin. J. Biol. Chem. 280: 34343-34349.

40. Mirza, M., S. Marston, et al. 2005. Dilated cardiomyopathy mutations in three thin filament regulatory proteins result in a common functional phenotype. J. Biol. Chem. 280: 28498-28506.

41. Kushwaha, S.S., J.T. Fallon \& V. Fuster. 1997. Restrictive cardiomyopathy. N. Engl. J. Med. 336: 267-276.

42. Mogensen, J., R. Kubo, et al. 2003. Idiopathic restrictive cardiomyopathy is part of the clinical expression of cardiac troponin I mutations. J. Clin. Invest. 111: 209-216.

43. Gomes, A.V., J. Liang \& J.D. PotTER. 2005. Mutations in human cardiac troponin I that are associated with restrictive cardiomyopathy affect basal ATPase activity and the calcium sensitivity of force development. J. Biol. Chem. 280: 3090930915.

44. Yumoto, F., Q.W. Lu, et al. 2005. Drastic Ca2+ sensitization of myofilament associated with a small structural change in troponin I in inherited restrictive cardiomyopathy. Biochem. Biophys. Res. Commun. 338: 1519-1526.

45. Haghighi, K., K.N. Gregory \& E.G. Kranias. 2004. Sarcoplasmic reticulum Ca-ATPase-phospholamban interactions and dilated cardiomyopathy. Biochem. Biophys. Res. Commun. 322: 1214-1222.

46. Szatkowski, M.L., M.V. Westfall, et al. 2001. In vivo acceleration of heart relaxation performance by Parvalbumin gene delivery. J. Clin. Invest. 107: 191198.

47. Rothermel, B.A., T.A. Mckinsey, R.B. Vega, et al. 2001. Myocyte-enriched calcineurin-interacting protein, MCIP1, inhibits cardiac hypertrophy in vivo. Proc. Natl. Acad. Sci. USA 98: 3328-3333.

48. Sussman, M.A., H.W. Lim, N. Gude, et al. 1998. Prevention of cardiac hypertrophy in mice by calcineurin inhibition. Science 281: 1690-1693.

49. ZhANG, R., M.S. KHOO, Y. Wu, et al. 2005. Calmodulin kinase II inhibition protects against structural heart disease. Nat. Med. 11: 409-417. 
50. RobBins, J. 2000. Remodeling the cardiac sarcomere using transgenesis. Annu. Rev. Physiol. 62: 261-287.

51. Michele, D.E., F. Albayya \& J.M. Metzger 1999. Thin filament protein dynamics in fully differentiated adult cardiac myocytes: toward a model of sarcomere maintenance. J. Cell. Biol. 145: 1483-1495. 